\title{
Porous Cyclotrimethylenetrinitramine with Reduced Sensitivity Prepared by a Solvation-Desolvation Method
}

Mi Yan, ${ }^{[a]}$ Yu Liu, ${ }^{[a]}$ Jinjiang Xu, ${ }^{[a]}$ Liyuan Yang, ${ }^{[a],}[\mathrm{b}]$ Lin Zhang, ${ }^{[a],}{ }^{[c]}$ Fude Nie, ${ }^{\text {[a] }}$ and Shiliang Huang ${ }^{*}[\mathrm{a}]$

\footnotetext{
${ }^{[a]}$ Institute of Chemical Materials, China Academy of Engineering Physics, Mianyang, 621900, PR China

${ }^{[b]}$ Luzhou North Chemical Industries Co., Ltd., China North Industries Group Corporation Limited, Luzhou, 646003, PR China

${ }^{[c]}$ School of Chemical Engineering and Technology, Tianjin University, Tianjin, 300072, PR China
} 


\section{List of Contents}

Figure S1. Optical microscopy images of RDX·TMS, RDX·CAM and RDX·HMPT crystals.

Figure S2. Experimental and simulated X-ray powder diffraction patterns of RDX·TMS.

Figure S3. Experimental and simulated X-ray powder diffraction patterns of RDX.CAM.

Figure S4. Experimental and simulated X-ray powder diffraction patterns of RDX·HMPT.

Figure S5. The configurations of the RDX molecule in (a) RDX·HMPT and (b) $\beta$-RDX.

Figure S6. TG-DSC measurement of RDX·TMS.

Figure S7. TG-DSC measurement of RDX.CAM.

Figure S8. The optical microscopy images of RDX·HMPT soaked in a drop of mother liquor after exposed to the air for different time.

Figure S9. Powder X-ray diffraction patterns of desolvated RDX·TMS (RDX-T $\left.\mathrm{H}_{2} \mathrm{O}\right)$, RDX.CAM (RDX-C $\left.\mathrm{H}_{2} \mathrm{O}\right)$ and RDX·HMPT (RDX- $\left.\mathrm{H}_{\mathrm{H}_{2} \mathrm{O}}\right)$ obtained by anti-solvent extraction.

Figure S10. $\mathrm{N}_{2}$ adsorption/desorption measurement of porous RDX transformed from RDX.CAM by anti-solvent extraction.

Figure S11. Pore size distribution of porous RDX transformed from RDX.CAM by antisolvent extraction.

Figure S12. TG-DSC measurement of RDX transformed from (a) RDX.TMS, (b) RDX.CAM and (c) RDX.HMPT by vacuum pyrolysis in nitrogen flow and a heat rate of $10{ }^{\circ} \mathrm{C} / \mathrm{min}$.

Figure S13. Mean particle sizes of $\mathrm{RDX}-\mathrm{T}_{\mathrm{H}_{2} \mathrm{O}}$ and $\mathrm{RDX}-\mathrm{C}_{\mathrm{H}_{2} \mathrm{O}}$ after ultrasonic treatment.

Table S1. The sensitivity test parameters.

Table S2. Hydrogen bond lengths $(\AA)$ and angles $\left(^{\circ}\right)$ in RDX·TMS, RDX·CAM and RDX·HMPT.

Table S3. The pore size of porous RDX-T $\mathrm{T}_{\text {vac. }}$ calculated by SEM images.

Table S4. The pore size of porous RDX-T $\mathrm{T}_{\mathrm{H} 2 \mathrm{O}}$ calculated by SEM images.

Table S5. The pore size of porous $\mathrm{RDX}-\mathrm{C}_{\mathrm{vac}}$. calculated by SEM images.

Table S6. The pore size of porous RDX- $\mathrm{C}_{\mathrm{H} 2 \mathrm{O}}$ calculated by SEM images.

Table S7. The pore size of porous RDX-Hvac. calculated by SEM images.

Table S8. The pore size of porous RDX-H $\mathrm{H}_{2} \mathrm{O}$ calculated by SEM images.

Table S9. The particle sizes of porous RDX after ultrasonic treated. 


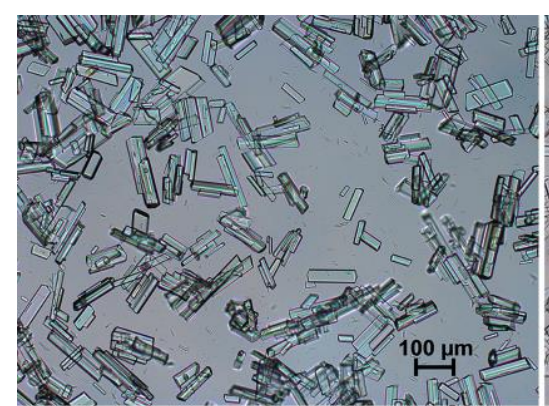

a

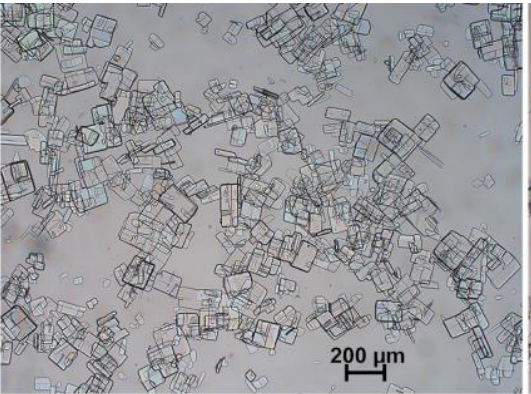

b

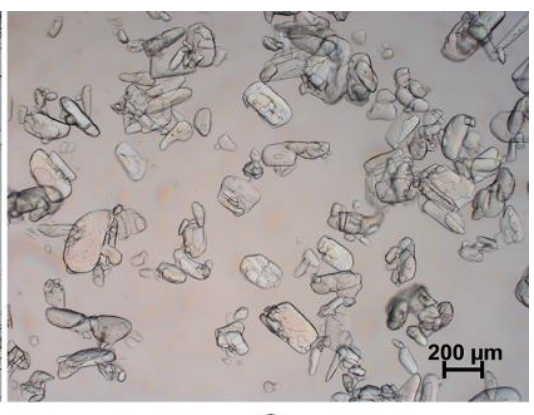

$\mathrm{C}$

Figure S1. Optical microscopy images of RDX·TMS, RDX·CAM and RDX·HMPT crystals.

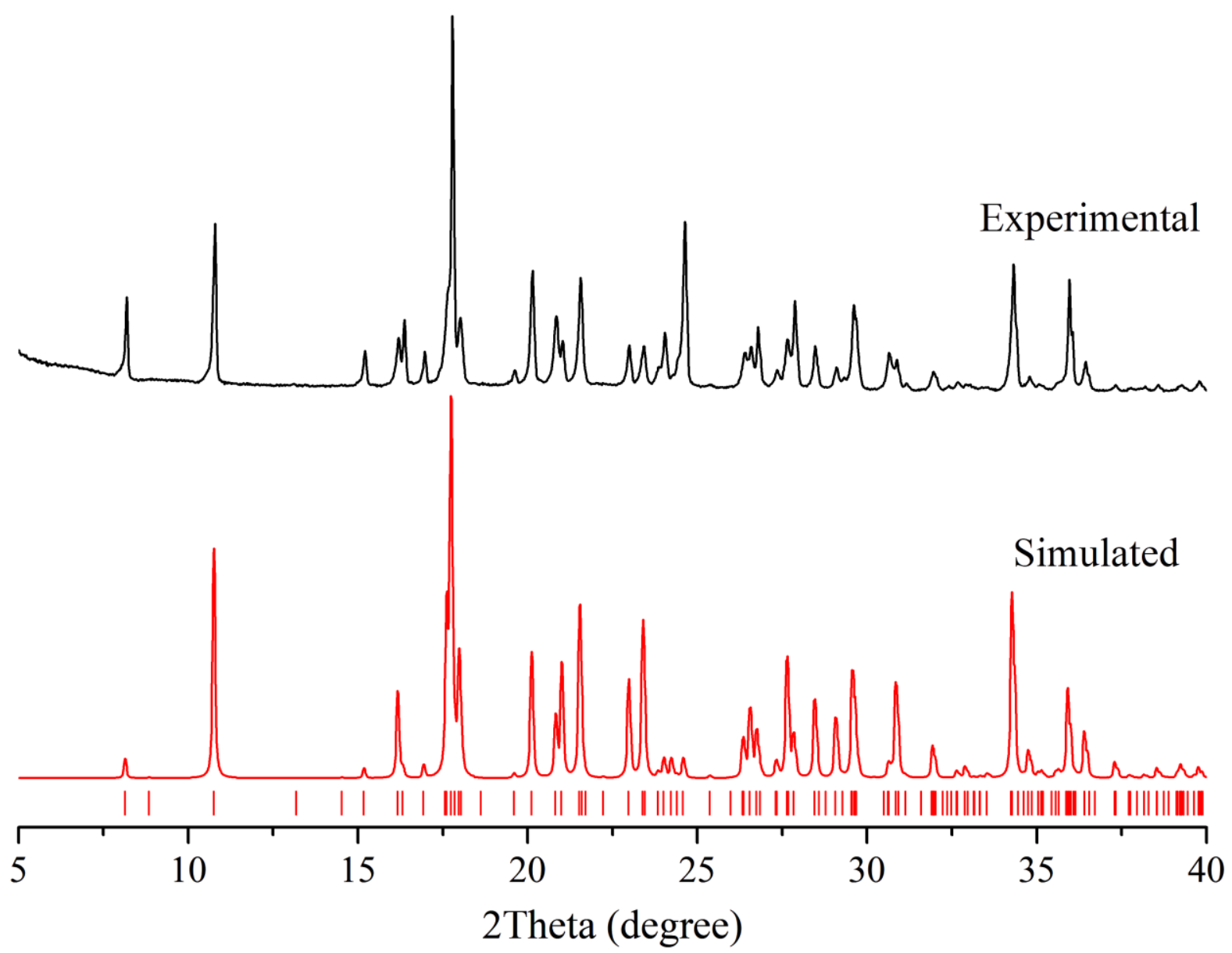

Figure S2. Experimental (black) X-ray powder diffraction patterns of RDX·TMS and that simulated from the structure model (red). Short vertical lines indicate calculated peak positions. Because the RDX·TMS crystal has plate-like morphology, preferred orientation along 010 and parameter $01=1.5$ o2 $=0$ were involved in the simulation in Powder Cell program (http://www.ccp14.ac.uk/ccp/web-mirrors/powdcell/a_v/v_1/powder/e_cell.html). 


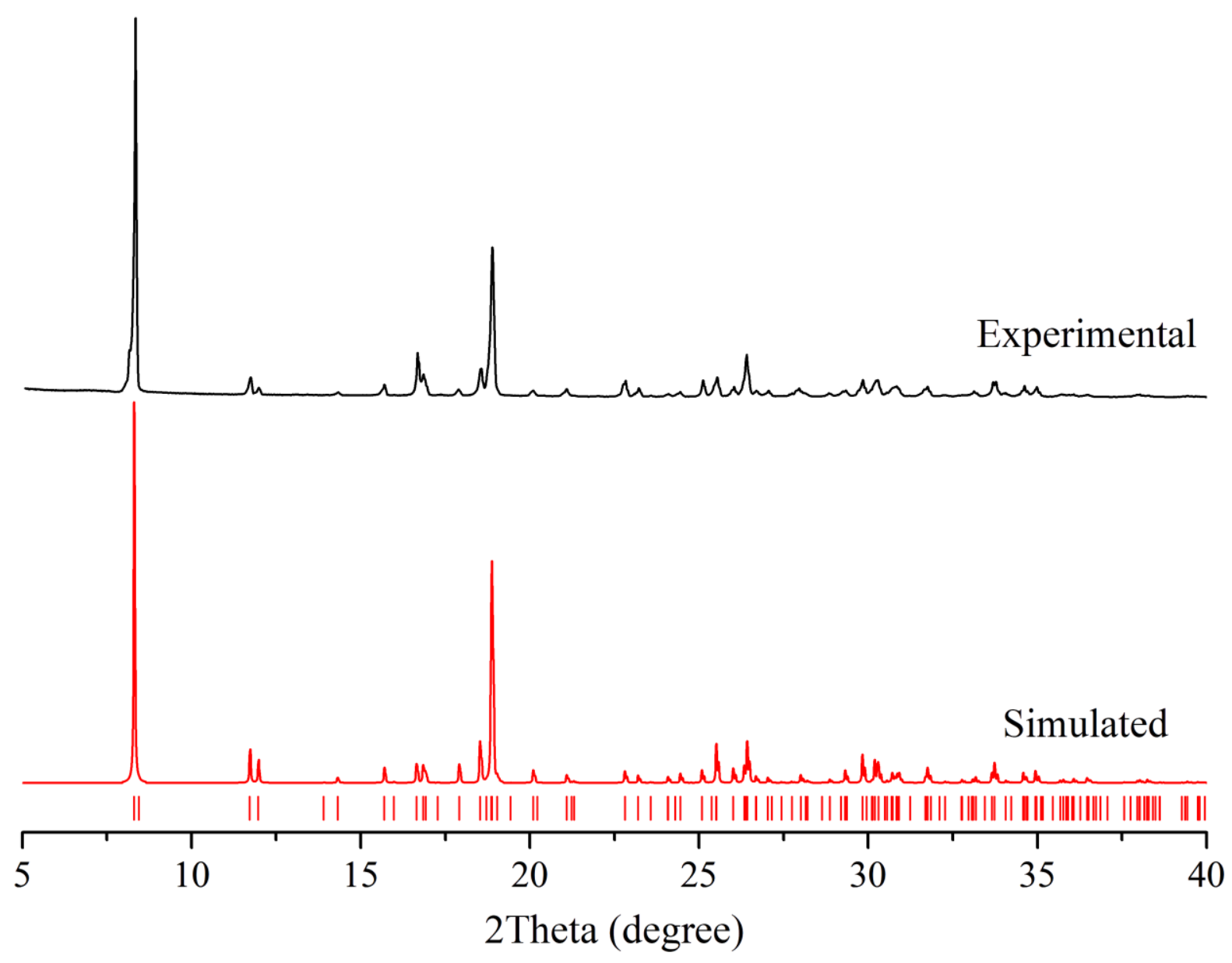

Figure S3. Experimental (black) X-ray powder diffraction patterns of RDX.CAM and that simulated from the structure model (red). Short vertical lines indicate calculated peak positions. Because the RDX·CAM crystal has plate-like morphology, preferred orientation along 001 and parameter $01=1.0$ o2=0 were involved in the simulation in Powder Cell program (http://www.ccp14.ac.uk/ccp/web-mirrors/powdcell/a_v/v_1/powder/e_cell.html). 


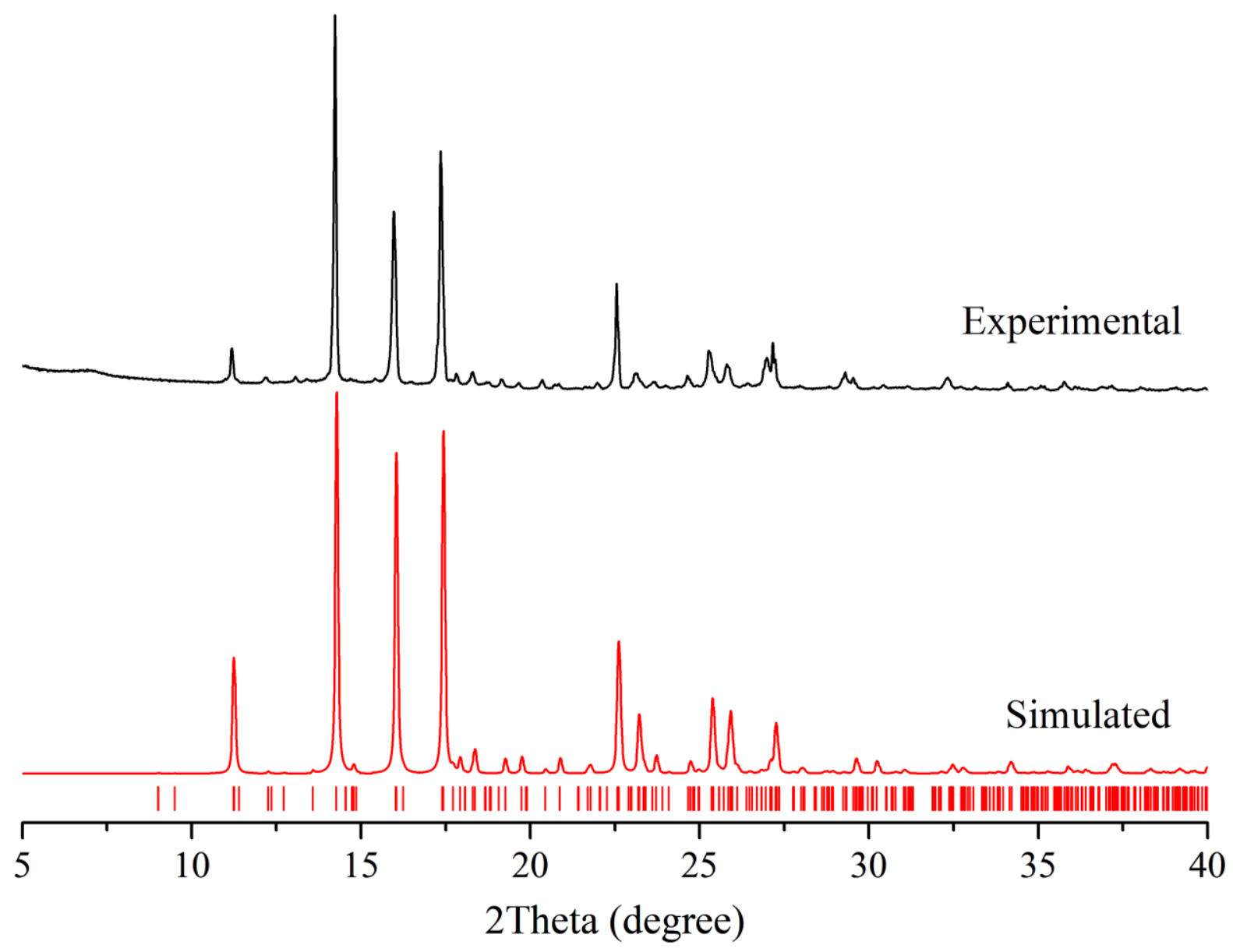

Figure S4. Experimental (black) X-ray powder diffraction patterns of RDX·HMPT and that simulated from the structure model (red). Short vertical lines indicate calculated peak positions. Because the RDX·HMPT crystal has needle-like morphology, preferred orientation along 001 and parameter $01=2.0$ o2 $=0$ were involved in the simulation in Powder Cell program (http://www.ccp14.ac.uk/ccp/web-mirrors/powdcell/a_v/v_1/powder/e_cell.html).
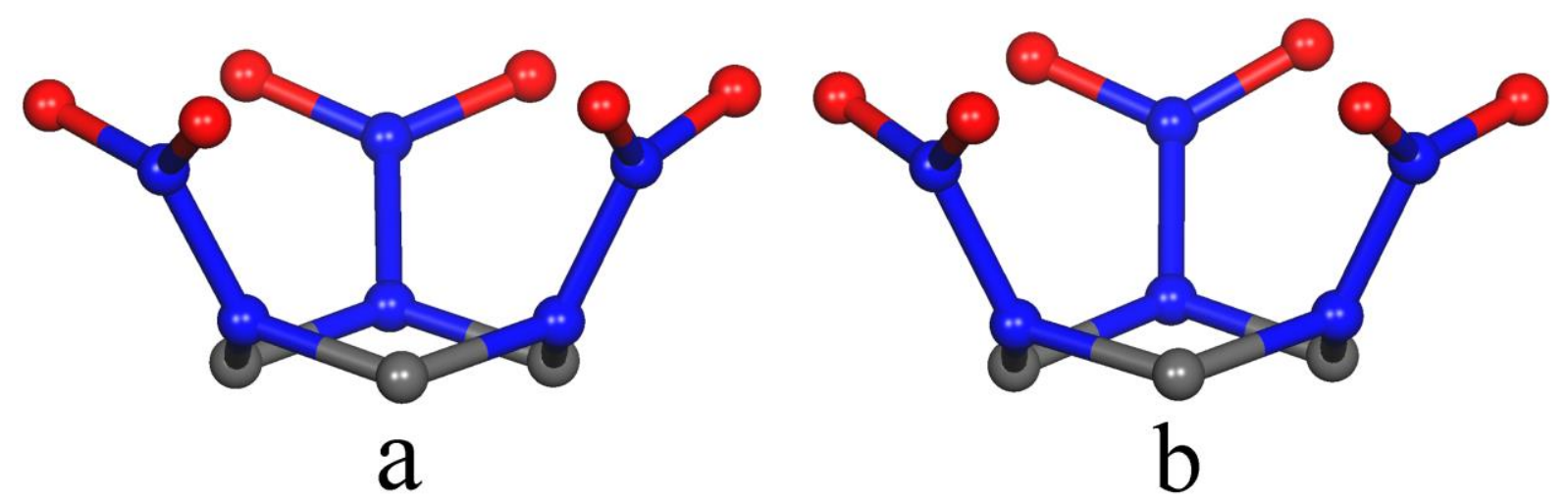

Figure S5. The configurations of the RDX molecule in (a) RDX·HMPT and (b) $\beta$-RDX. 


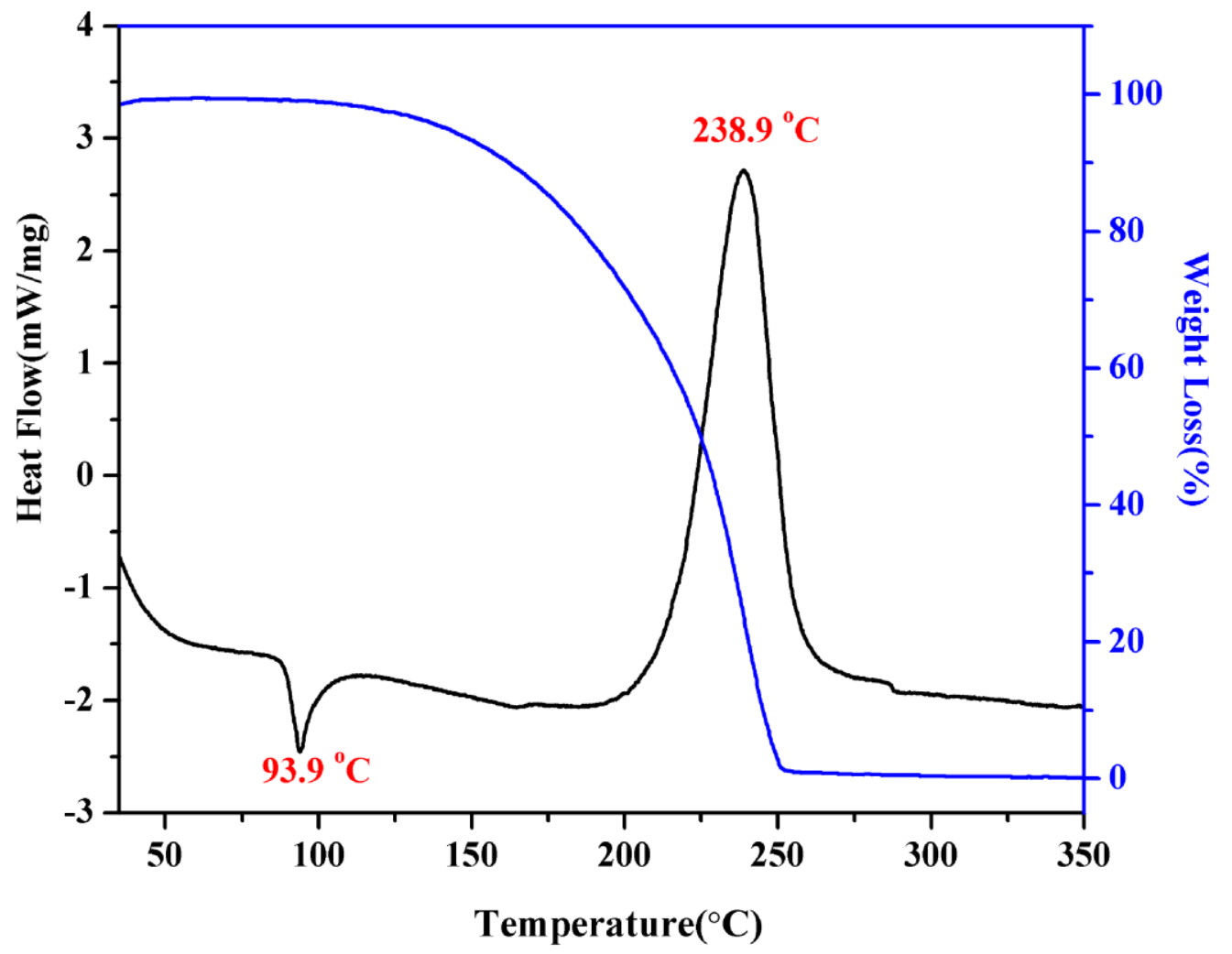

Figure S6. TG-DSC measurement of RDX.TMS in nitrogen flow and a heat rate of 10 ${ }^{\circ} \mathrm{C} / \mathrm{min}$.

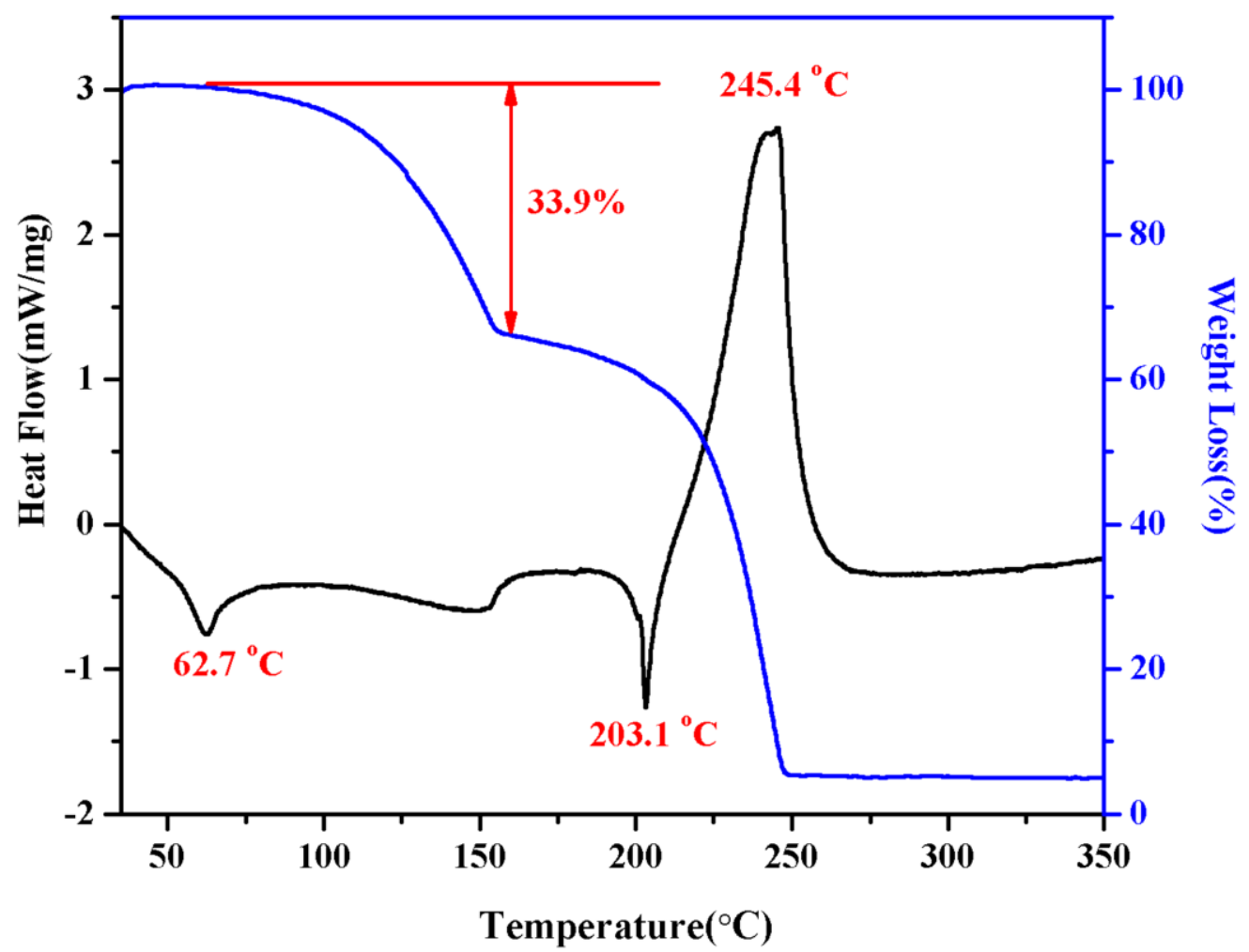

Figure S7. TG-DSC measurement of RDX.CAM in nitrogen flow and a heat rate of 10 ${ }^{\circ} \mathrm{C} / \mathrm{min}$. 


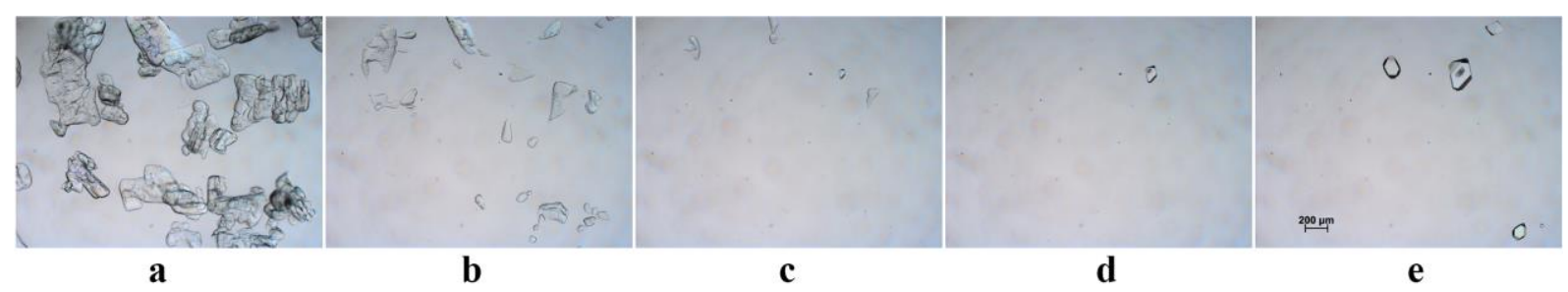

Figure S8. The optical microscopy images of RDX.HMPT soaked in a drop of mother liquor after exposed to the air for different time (a. 5 minutes, b. 15 minutes, c. 25 minutes, d. 35minutes, d. 45 minutes).

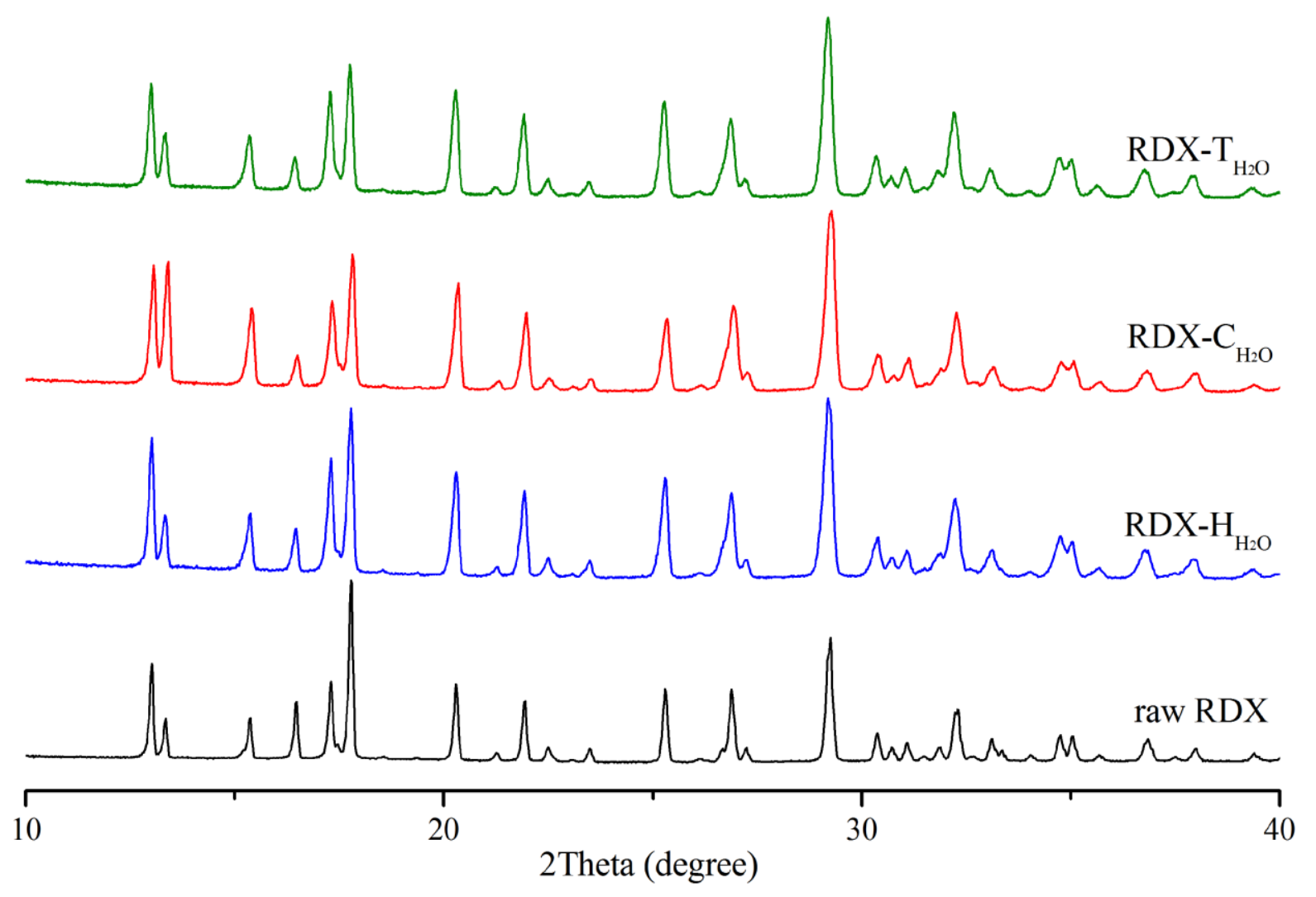

Figure S9. Powder X-ray diffraction patterns of desolvated RDX-TMS (RDX-T $\mathrm{H}_{2} \mathrm{O}$ ), RDX.CAM (RDX- $\left.\mathrm{C}_{2} \mathrm{O}\right)$ and $\mathrm{RDX} \cdot \mathrm{HMPT}\left(\mathrm{RDX}-\mathrm{H}_{\mathrm{H}_{2} \mathrm{O}}\right)$ obtained by anti-solvent extraction and raw RDX. 


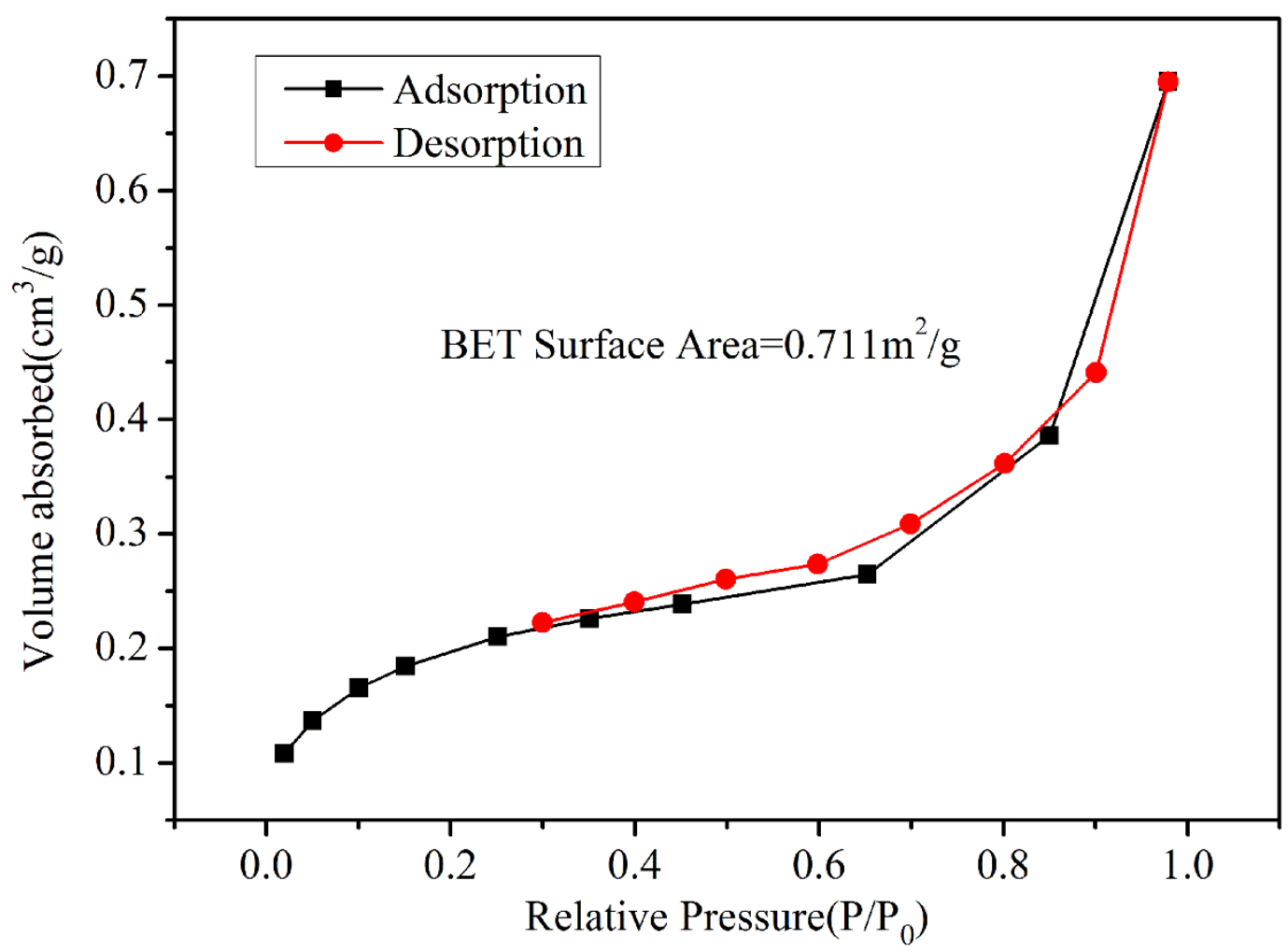

Figure S10. $\mathrm{N}_{2}$ adsorption/desorption measurement of porous RDX transformed from RDX.CAM by anti-solvent extraction.

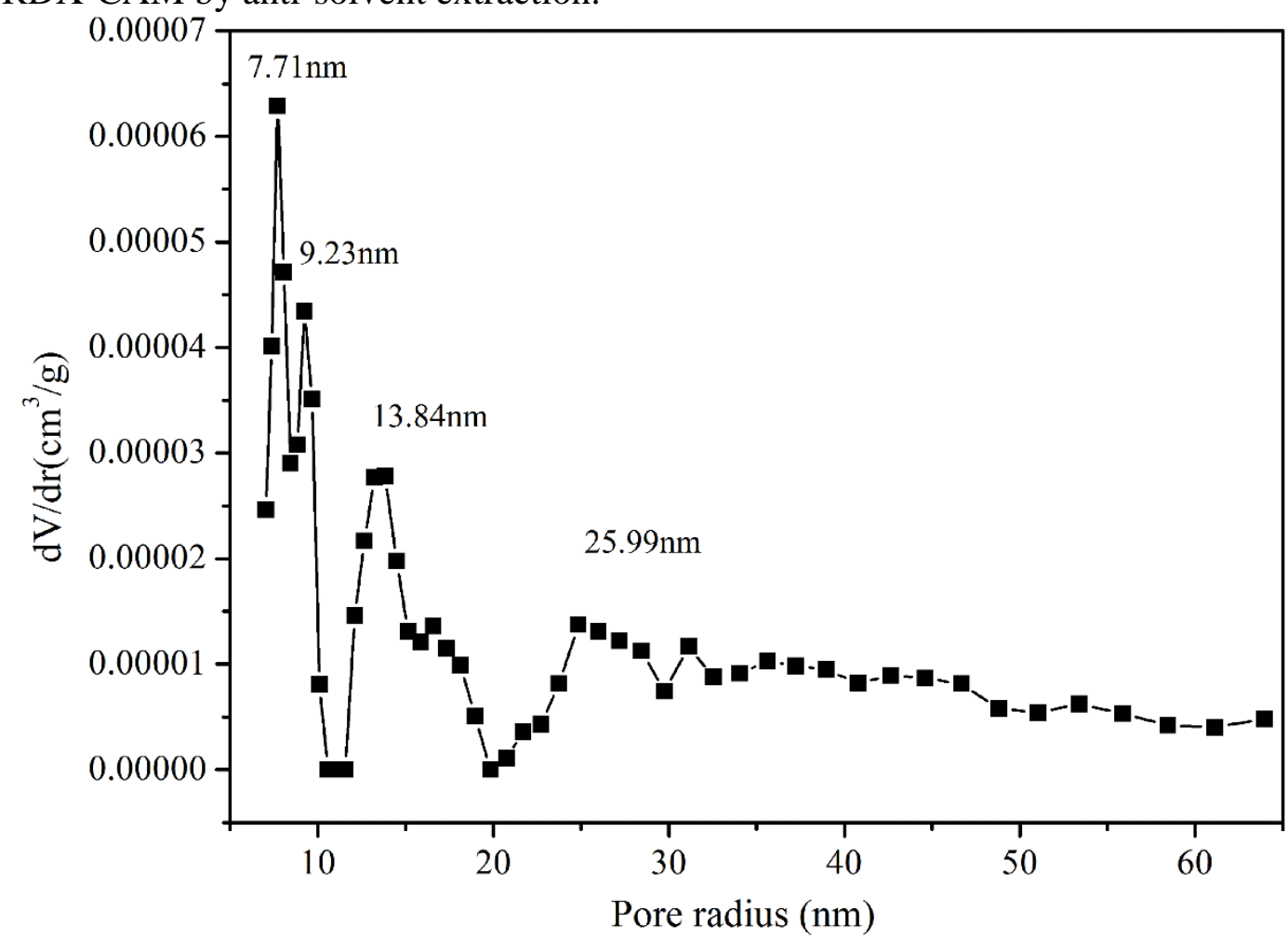

Figure S11. Pore size distribution of porous RDX transformed from RDX.CAM by antisolvent extraction. 
Supplementary information
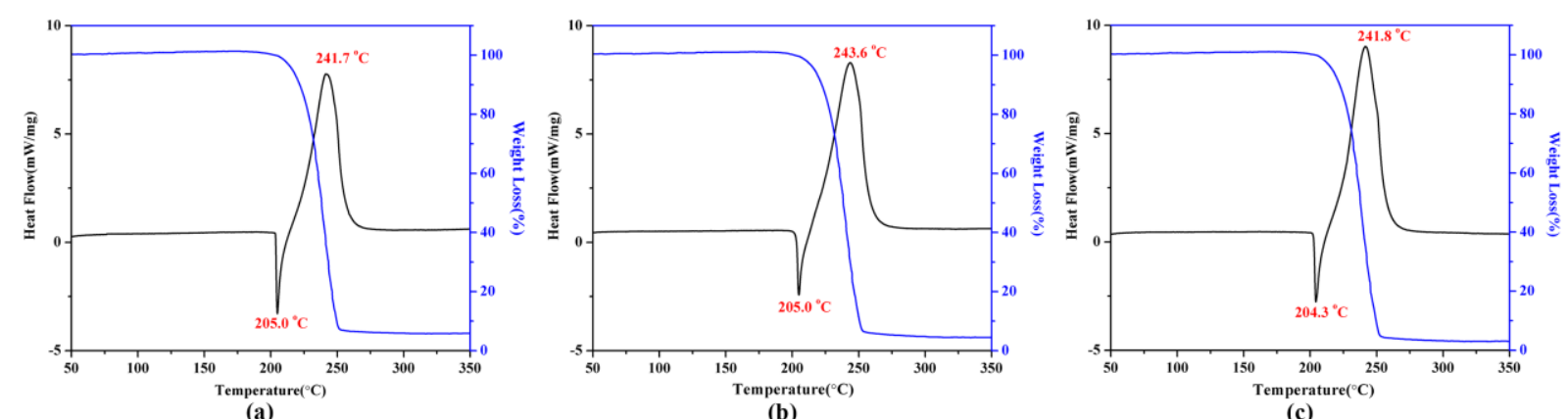

Figure S12. TG-DSC measurement of RDX transformed from (a) RDX·TMS, (b) RDX.CAM and (c) RDX.HMPT by vacuum pyrolysis in nitrogen flow and a heat rate of $10{ }^{\circ} \mathrm{C} / \mathrm{min}$.
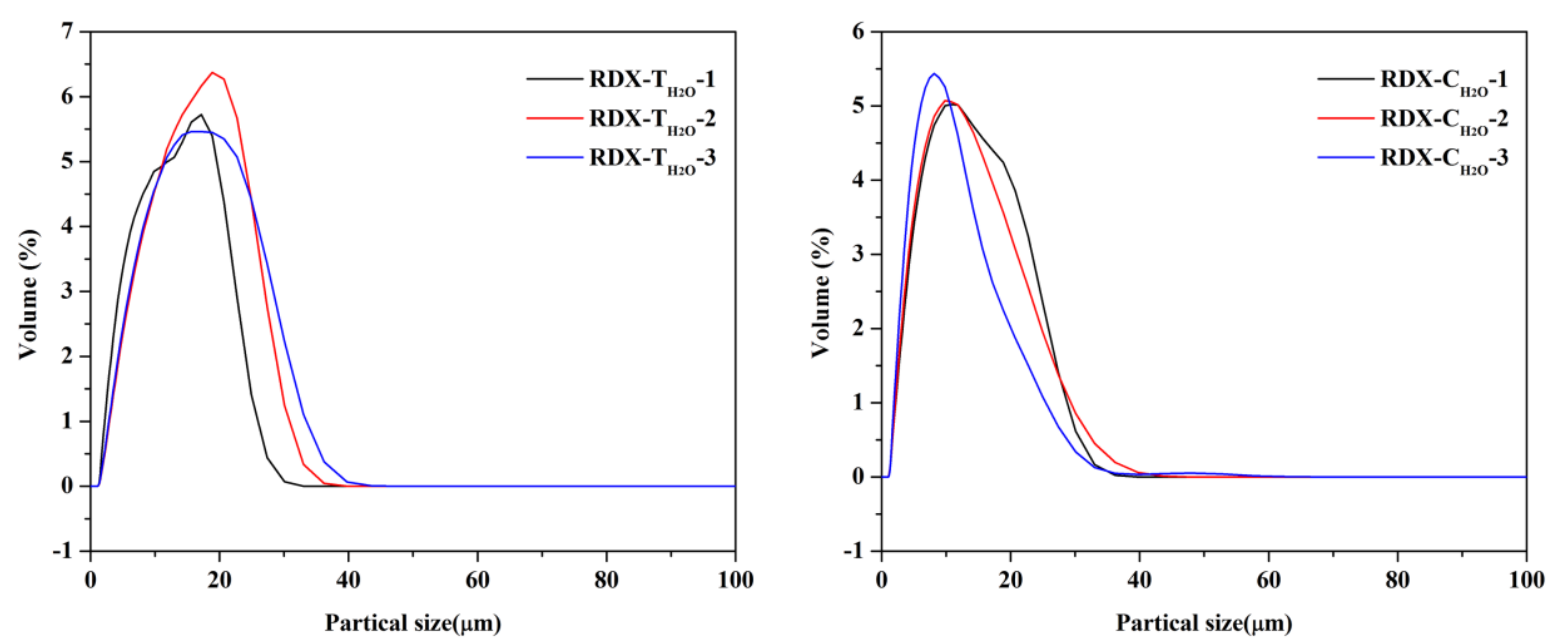

Figure S13. Mean particle sizes of RDX- $\mathrm{T}_{\mathrm{H} 2 \mathrm{O}}$ and $\mathrm{RDX}-\mathrm{C}_{\mathrm{H} 2 \mathrm{O}}$ after ultrasonic treatment. 
Table S1. The sensitivity test parameters.

\begin{tabular}{l|c|c|c|c|c|c}
\hline \multirow{2}{*}{$\begin{array}{l}\text { Impact } \\
\text { sensitivity }\end{array}$} & $\begin{array}{c}\text { Sample mass } \\
(\mathrm{mg})\end{array}$ & $\begin{array}{c}\text { Drop weight } \\
\text { mass } \\
(\mathrm{kg})\end{array}$ & $\begin{array}{c}\text { Drop weight height } \\
(\mathrm{cm})\end{array}$ & $\begin{array}{c}\text { Ambient } \\
\text { temperature } \\
\left({ }^{\circ} \mathrm{C}\right)\end{array}$ & $\begin{array}{c}\text { Ambient } \\
\text { humidity } \\
(\%)\end{array}$ \\
\cline { 2 - 7 } $\begin{array}{l}\text { Friction } \\
\text { sensitivity }\end{array}$ & $\begin{array}{c}\text { Sample mass } \\
(\mathrm{mg})\end{array}$ & $\begin{array}{c}\text { Weight } \\
\text { mass } \\
(\mathrm{kg})\end{array}$ & $\begin{array}{c}\text { Pressure } \\
(\mathrm{MPa})\end{array}$ & $\begin{array}{c}\text { Swing } \\
\text { angle } \\
\left({ }^{\circ}\right)\end{array}$ & $\begin{array}{c}\text { Ambient } \\
\text { temperature } \\
\left({ }^{\circ} \mathrm{C}\right)\end{array}$ & $\begin{array}{c}\text { Ambient } \\
\text { humidity } \\
(\%)\end{array}$ \\
\cline { 2 - 8 } & 20 & 1.5 & 3.92 & 90 & 24 & 71 \\
\hline $\begin{array}{l}\text { Electrostatic } \\
\text { spark } \\
\text { sensitivity }\end{array}$ & $\begin{array}{c}\text { Sample mass } \\
(\mathrm{mg})\end{array}$ & pole & $\begin{array}{c}\text { Capacity } \\
(\mathrm{pF})\end{array}$ & $\begin{array}{c}\text { Electrode } \\
\text { interval }(\mathrm{mm})\end{array}$ & $\begin{array}{c}\text { Ambient } \\
\text { temperature } \\
\left({ }^{\circ} \mathrm{C}\right)\end{array}$ & $\begin{array}{c}\text { Ambient } \\
\text { humidity } \\
(\%)\end{array}$ \\
\cline { 2 - 8 } & 20 & - & 30000 & 0.5 & 25 & 62 \\
\hline
\end{tabular}

Table S2. Hydrogen bond lengths $(\AA)$ and angles $\left(^{\circ}\right)$ in RDX·TMS, RDX.CAM and RDX·HMPT.

\begin{tabular}{|c|c|c|c|c|}
\hline RDX/TMS & $\mathbf{D}-\mathbf{H} \cdots \mathbf{A}$ & $\mathbf{d}(\mathbf{H} \cdots \mathbf{A})$ & $d(D \cdots A)$ & $\angle \mathrm{DHA}$ \\
\hline & $\mathrm{C} 1-\mathrm{H} 1 \mathrm{~A} \cdots \mathrm{O} 8$ & 2.598 & 3.245 & 124.376 \\
\hline & $\mathrm{C} 2-\mathrm{H} 2 \mathrm{~B} \cdots \mathrm{O} 7$ & 2.410 & 3.292 & 150.990 \\
\hline & $\mathrm{C} 6-\mathrm{H} 6 \mathrm{~B} \cdots \mathrm{O} 1$ & 2.578 & 3.446 & 149.036 \\
\hline \multirow[t]{3}{*}{ RDX·CAM } & $\mathbf{D}-\mathbf{H} \cdots \mathbf{A}$ & $\mathbf{d}(\mathbf{H} \cdots \mathbf{A})$ & $d(D \cdots A)$ & $\angle$ DHA \\
\hline & $\mathrm{C} 2-\mathrm{H} 2 \mathrm{~B} \cdots \mathrm{O} 7$ & 2.441 & 3.269 & 143.142 \\
\hline & $\mathrm{C} 3-\mathrm{H} 3 \mathrm{~A} \cdots \mathrm{O} 7$ & 2.361 & 3.248 & 151.744 \\
\hline \multirow[t]{10}{*}{ RDX·HMPT } & $\mathbf{D}-\mathbf{H} \cdots \mathbf{A}$ & $\mathbf{d}(\mathbf{H} \cdots \mathbf{A})$ & $d(D \cdots A)$ & $\angle$ DHA \\
\hline & $\mathrm{C} 1-\mathrm{H} 1 \mathrm{~B} \cdots \mathrm{O} 19$ & 2.373 & 3.128 & 134.223 \\
\hline & $\mathrm{C} 2-\mathrm{H} 2 \mathrm{~A} \cdots \mathrm{O} 19$ & 2.434 & 3.161 & 131.474 \\
\hline & $\mathrm{C} 3-\mathrm{H} 3 \mathrm{~B} \cdots \mathrm{O} 19$ & 2.408 & 3.152 & 133.111 \\
\hline & $\mathrm{C} 4-\mathrm{H} 4 \mathrm{~B} \cdots \mathrm{O} 20$ & 2.464 & 3.179 & 130.246 \\
\hline & $\mathrm{C} 5-\mathrm{H} 5 \mathrm{~A} \cdots \mathrm{O} 20$ & 2.438 & 3.160 & 130.999 \\
\hline & $\mathrm{C} 6-\mathrm{H} 6 \mathrm{~B} \cdots \mathrm{O} 20$ & 2.396 & 3.145 & 133.743 \\
\hline & $\mathrm{C} 7-\mathrm{H} 7 \mathrm{~B} \cdots \mathrm{O} 21$ & 2.414 & 3.157 & 133.090 \\
\hline & $\mathrm{C} 8-\mathrm{H} 8 \mathrm{~B} \cdots \mathrm{O} 21$ & 2.377 & 3.128 & 133.970 \\
\hline & $\mathrm{C} 9-\mathrm{H} 9 \mathrm{~B} \cdots \mathrm{O} 21$ & 2.398 & 3.146 & 133.502 \\
\hline
\end{tabular}

Table S3. The pore size of porous RDX-T $\mathrm{T}_{\mathrm{vac}}$. calculated by SEM images.

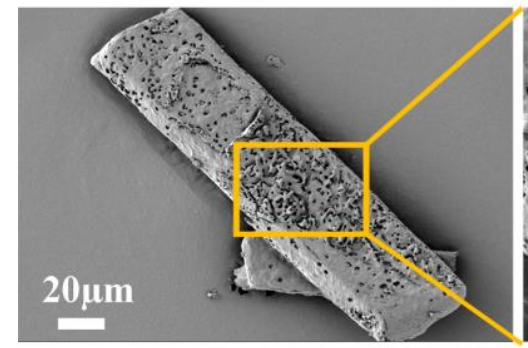

a

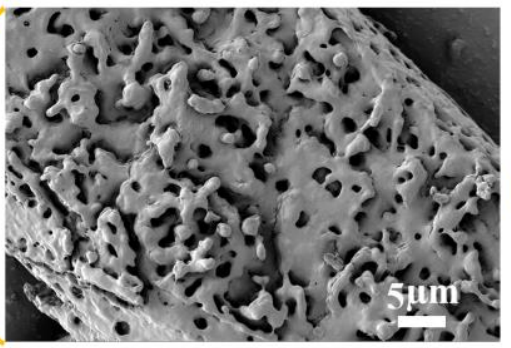

b

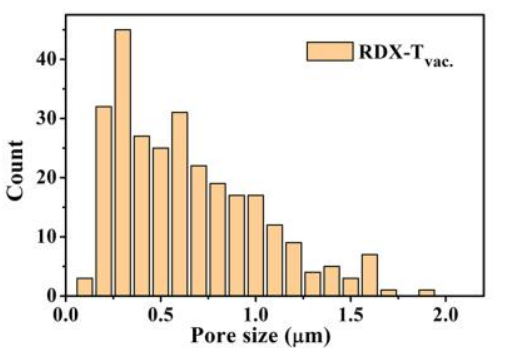

C

\begin{tabular}{|c|c|c|c|c|c|c|c|c|c|c|c|c|c|c|c|c|c|c|c|c|}
\hline RDX-Tvac. & Bin End $(\mu \mathrm{m})$ & 0.1 & 0.2 & 0.3 & 0.4 & 0.5 & 0.6 & 0.7 & 0.8 & 0.9 & 1 & 1.1 & 1.2 & 1.3 & 1.4 & 1.5 & 1.6 & 1.7 & 1.8 & 1.9 \\
\hline & Count & 3 & 32 & 45 & 27 & 25 & 31 & 22 & 19 & 17 & 17 & 12 & 9 & 4 & 5 & 3 & 7 & 1 & 0 & 1 \\
\hline
\end{tabular}


Table S4. The pore size of porous RDX- $\mathrm{T}_{\mathrm{H} 2 \mathrm{O}}$ calculated by SEM images.

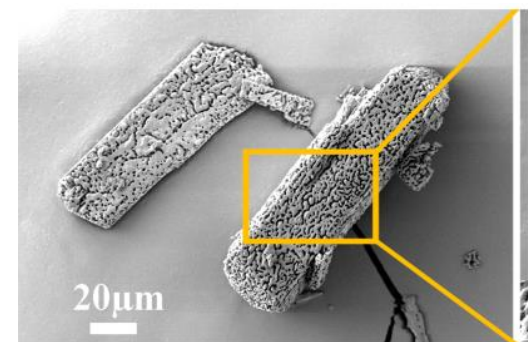

a

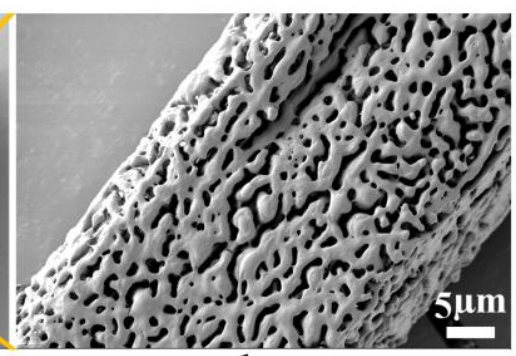

b

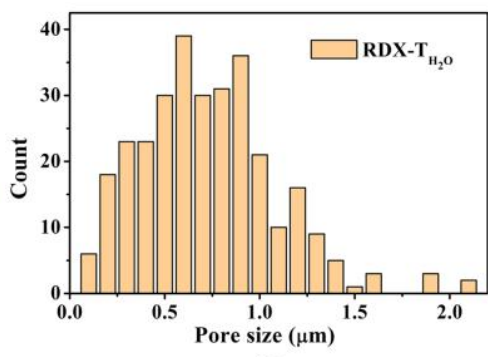

C

\begin{tabular}{|c|c|c|c|c|c|c|c|c|c|c|c|c|c|c|c|c|c|c|c|c|c|c|}
\hline RDX-Tн2о & Bin End $(\mu \mathrm{m})$ & 0.1 & 0.2 & 0.3 & 0.4 & 0.5 & 0.6 & 0.7 & 0.8 & 0.9 & 1 & 1.1 & 1.2 & 1.3 & 1.4 & 1.5 & 1.6 & 1.7 & 1.8 & 1.9 & 2 & 2.1 \\
\hline & Count & 6 & 18 & 23 & 23 & 30 & 39 & 30 & 31 & 36 & 21 & 10 & 16 & 9 & 5 & 1 & 3 & 0 & 0 & 3 & 0 & 2 \\
\hline
\end{tabular}

Table S5. The pore size of porous RDX-C $\mathrm{C}_{\mathrm{vac}}$. calculated by SEM images.

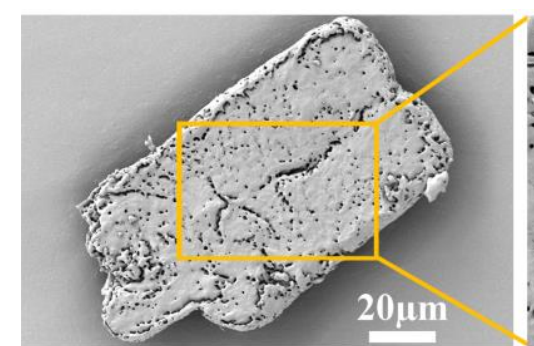

a

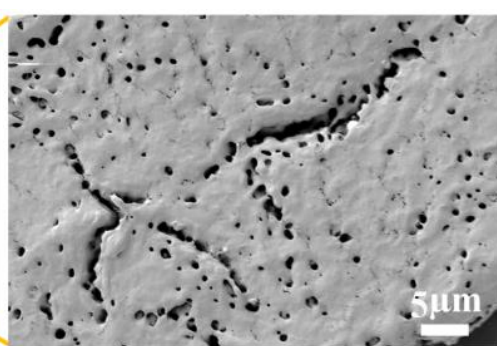

b

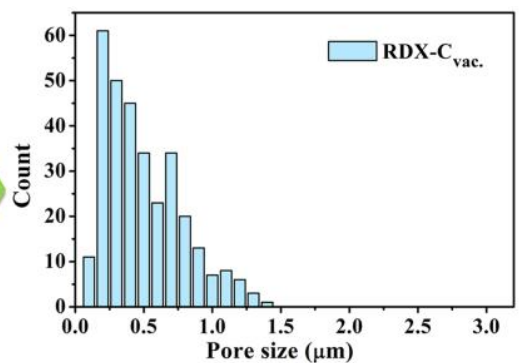

C

\begin{tabular}{|c|c|c|c|c|c|c|c|c|c|c|c|c|c|c|c|}
\hline RDX-Cvac. & Bin End $(\mu \mathrm{m})$ & 0.1 & 0.2 & 0.3 & 0.4 & 0.5 & 0.6 & 0.7 & 0.8 & 0.9 & 1 & 1.1 & 1.2 & 1.3 & 1.4 \\
\hline & Count & 11 & 61 & 50 & 45 & 34 & 23 & 34 & 20 & 13 & 7 & 8 & 6 & 3 & 1 \\
\hline
\end{tabular}

Table S6. The pore size of porous RDX- $\mathrm{C}_{\mathrm{H} 2 \mathrm{O}}$ calculated by SEM images.

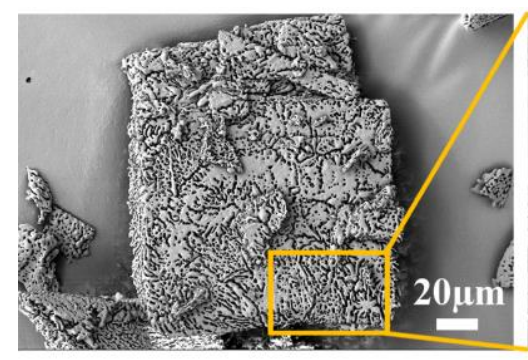

a

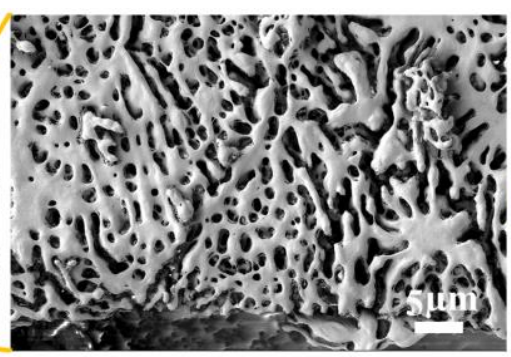

b

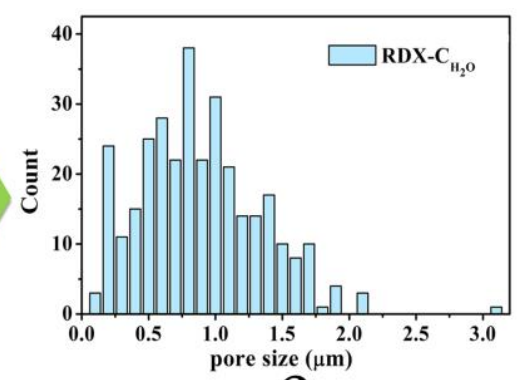

C

\begin{tabular}{|c|c|c|c|c|c|c|c|c|c|c|c|c|c|c|c|c|c|}
\hline RDX-C & Bin End $(\mu \mathrm{m})$ & 0.1 & 0.2 & 0.3 & 0.4 & 0.5 & 0.6 & 0.7 & 0.8 & 0.9 & 1 & 1.1 & 1.2 & 1.3 & 1.4 & 1.5 & 1.6 \\
\hline & Count & 3 & 24 & 11 & 15 & 25 & 28 & 22 & 38 & 22 & 31 & 21 & 14 & 14 & 17 & 10 & 8 \\
\hline & & 1.7 & 1.8 & 1.9 & 2 & 2.1 & 2.2 & 2.3 & 2.4 & 2.5 & 2.6 & 2.7 & 2.8 & 2.9 & 3 & 3.1 & \\
\hline & & 10 & 1 & 4 & 0 & 3 & 0 & 0 & 0 & 0 & 0 & 0 & 0 & 0 & 0 & 1 & \\
\hline
\end{tabular}


Table S7. The pore size of porous RDX-Hvac. calculated by SEM images.

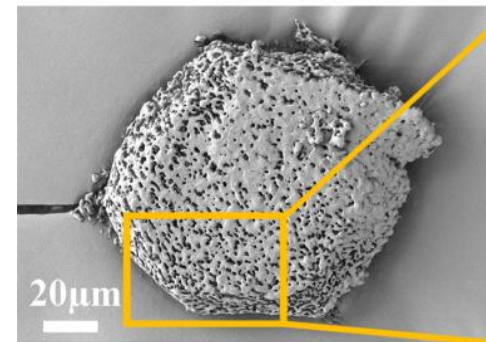

a

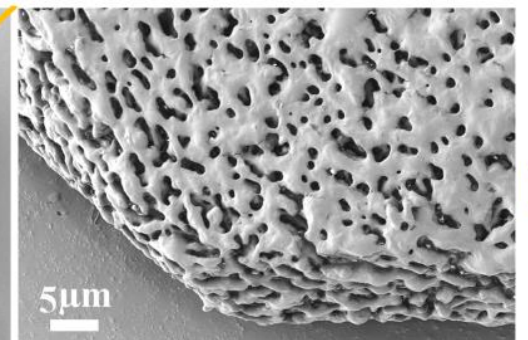

b

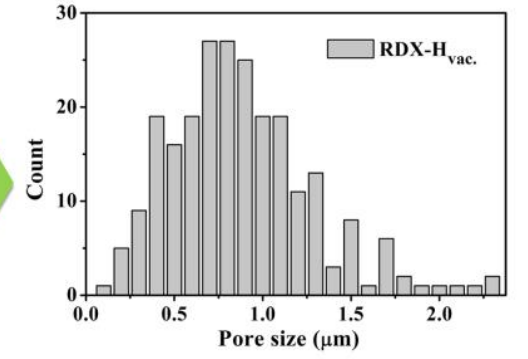

C

\begin{tabular}{|c|c|c|c|c|c|c|c|c|c|c|c|c|c|}
\hline RDX-Hvac & Bin End $(\mu \mathrm{m})$ & 0.1 & 0.2 & 0.3 & 0.4 & 0.5 & 0.6 & 0.7 & 0.8 & 0.9 & 1 & 1.1 & 1.2 \\
\hline & Count & 1 & 5 & 9 & 19 & 16 & 19 & 27 & 27 & 25 & 19 & 19 & 11 \\
\hline & & 1.3 & 1.4 & 1.5 & 1.6 & 1.7 & 1.8 & 1.9 & 2 & 2.1 & 2.2 & 2.3 & 2.4 \\
\hline & & 13 & 3 & 8 & 1 & 6 & 2 & 1 & 1 & 1 & 1 & 2 & 0 \\
\hline
\end{tabular}

Table S8. The pore size of porous RDX- $\mathrm{H}_{\mathrm{H} 2 \mathrm{O}}$ calculated by SEM images.

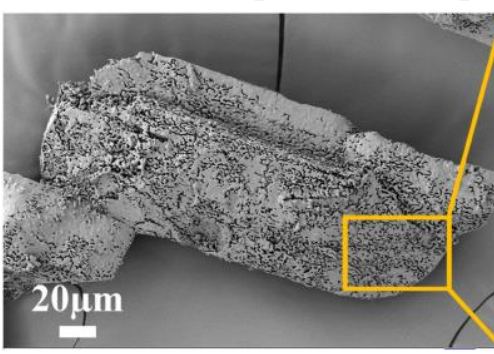

a

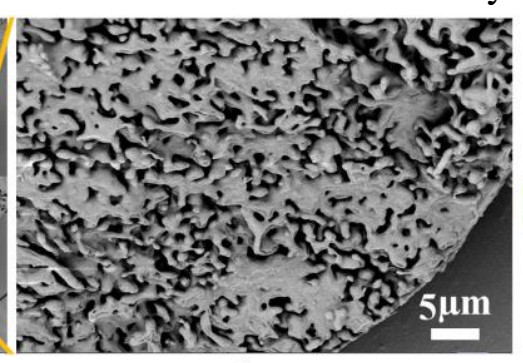

b

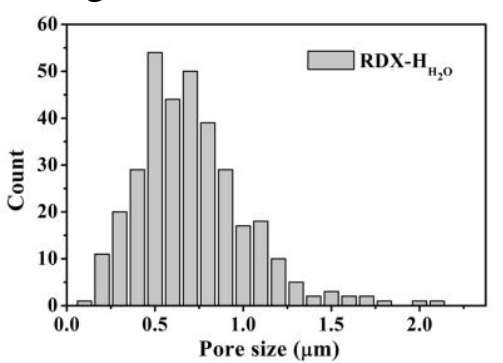

C

\begin{tabular}{|l|c|c|c|c|c|c|c|c|c|c|c|c|c|c|c|c|c|c|c|c|c|c|c|}
\hline RDX-HH2O & \multicolumn{2}{|c|}{ Bin End $(\mu \mathrm{m})$} & 0.1 & 0.2 & 0.3 & 0.4 & 0.5 & 0.6 & 0.7 & 0.8 & 0.9 & 1 & 1.1 & 1.2 & 1.3 & 1.4 & 1.5 & 1.6 & 1.7 & 1.8 & 1.9 & 2 & 2.1 \\
\hline & Count & 1 & 11 & 20 & 29 & 54 & 44 & 50 & 39 & 29 & 17 & 18 & 10 & 5 & 2 & 3 & 2 & 2 & 1 & 0 & 1 & 1 \\
\hline
\end{tabular}

Table S9. The particle sizes of porous RDX after ultrasonic treated.

\begin{tabular}{|c|c|c|c|c|}
\hline & \multicolumn{4}{|c|}{ Particle Size $(\mu \mathrm{m})$} \\
\cline { 2 - 5 } & Average & $\mathrm{d} 50$ & $\mathrm{~d} 10$ & $\mathrm{~d} 90$ \\
\hline RDX-T $\mathrm{H}_{2} \mathrm{O}-1$ & 14.1 & 12.8 & 4.5 & 25.8 \\
\hline RDX-T $\mathrm{H}_{2} \mathrm{O}-2$ & 13.9 & 13.1 & 4.7 & 24.5 \\
\hline RDX-T & 11.2 & 10.0 & 3.6 & 20.4 \\
\hline RDX- $\mathrm{H}_{2} \mathrm{O}-3$ & 11.0 & 9.3 & 3.4 & 21.1 \\
\hline RDX- $\mathrm{C}_{\mathrm{H} 2} \mathrm{O}-2$ & 11.2 & 9.7 & 3.5 & 21.6 \\
\hline RDX- $\mathrm{C}_{22} \mathrm{O}-3$ & 11.7 & 10.5 & 3.8 & 21.6 \\
\hline
\end{tabular}

